\title{
Diagnóstico ultrasonográfico antenatal y cirugía de malformaciones urológicas en recién nacidos
}

\author{
Dr. Francisco Ossandón C. 3: Dr. Sergio Reinoso S.2; Dr. Claus Krebs W. 3 \\ Dr. Gabriel Martínez D. 3 : Dr. Arrigo Giuliano B. ${ }^{3}$ \\ Prenatal ultrasonographic diagnosis and surgical resolution \\ of urologic malformations in the newborn
}

\begin{abstract}
Ten cases of prenatal ultrasound diagnosis of urological malformations and their posterior surgical managment are presented. Prenatal ulirasound, was very accurate for the diagnosis of obstruction, but not in the identification of its actual cause. Survival results in the treatment of ureteropelvic junction obstruction (6 kidneys) were good and nephrostony was not necessary. One kidney with preoperative exclusion got better after pyeloplasty. Good results were also obtained in other procedures: nephroureterectomy and section of the bridge in a patient with horseshoe kidney, one case of inferior heminephrectomy and another of superior heminephrectomy. Recurrent prolapse in a child with temporary Blocksom vesicostony needed further surgery. No operation was required in a case of bilateral hydronephrosis caused by a distal rectal pouch in a patient with imperforate anus.
\end{abstract}

(Key words: urinary tract, anomalles, congenital, prenatal ultrasound diagnosis).

Con la introducción del ultrasonido (ecografía) en el estudio del feto in útero, la cirugía del

1. Departamento de Pediatria y Cirugía Infantil, División Ciencias Médicas Occidente, Facultad de Medicina, Universidad de Chile. Hospital San Juan de Dios.

2. Departamento de Pediatría y Cirugía Infantil, Unjversidad de la Frontera, Hospital Regional de Temuco.

3. Servicios de Cirugia Infantik, Pediatría, Obstetricia y Ginecología, Hospital Las Higueras, Talcahuano. recién nacido ( $\mathrm{RN}$ ) ha experimentado un gran progreso en el diagnóstico antenatal de malformaciones corregibles, especialmente renales o de la vía urinaria. Muchas de estas enfermedades se diagnosticaban tardiamente o pasaban desapercibidas para dar sintomas a edades mayores. Con la cirugía más precoz se evita el deterioro progresivo del rifín y la vía urinaria, permitiendo un mejor desarrollo de ellos.

Las malfonmaciones de la vía urinaria son muy sensibles al diagnóstico ecografico especialmente 
si tienen obstrucción o una masa renal. Asimismo se pueden detectar aquellas malformaciones que cursan con dilatación de la vía urinaria y un gran residuo vesical (ej. reflujo vésicoureteral masivo, síndrome del "prunebelly" $1,2,6$ ).

\section{MATERIAL Y METODO}

Se revisaron 10 casos con diagnóstico antenatal de malformaciones urológica.j, entre los años 1981-1985 en los hospitales Regional de Temuco y Las Higueras de Talcahuano, que resultaron como hallazgo de examen en ecografias de rutuna en el tercer trimestre del embarazo.

Los casos fueron identificados empleando ecógrafos de riempo real: ADR con transtuctor lineal de $\hat{\mathbf{s}, 5} \mathbf{M H}$. y sectorial Philips con transductor de $3 \mathrm{MHz}$.

Los criterios ecograficos empleados en el diagnóstico de las malformaciones fucron los habitualmente conocidos para este método de examen7.a: en el riñón la obstrucción se manifiesta por una sombra ecolransparente (orina acumulada) que scpara los ecos centrales que son muy ecuretringente: (paredes calicilarest. Ello permite ver meior el cortorno calicilar. cortical y medular renal También se puede delinitas pelvis y a veces uríter ya que la orina acumulada contrasta con la pared ureteral o pelvica $y^{\prime}$ tejidos adyacentes secorrefringente i. La observación de la vejica permite evaluar su forma. grosor de pared. tamaño $y$ frecuencia de vaciamicrto. En obstrucciones uretrales es posible encontrar la vejiga distendida. de partides engrosadas, siempre llena, que puede acompanarse de dilatación ureteral. pélvica y calicilar uni o bilateral, además de alteraciones corticales renales que se manifiestam an mayor ecorrefringencia de sli pared y adclgazaniento de ella com un ribión generalmente aumentado de volumen. La detección de oligohidroamnios en la ecografía materna refleja baja producción de orina tital con función renal muy comprometida.

Todos los recién nacidos de este estudio fueron manejados por la unidad de nefruurología infantil del Hospital Regjonal de Tenuco. donde se practicaron los exámenes complementarios necesarios para establecer el diagnóstico definitivo (pielografía, uretrocistografía. ecolomografía abdominal del RN) y se operaron los que necesitaron correcciones quirúrgicas.

\section{RESULTADOS}

En la ecografia antenatal se observaron imágenes sospechosas de obstrucción en 9 casos (figura 1) y de tumor en 1 caso. Se confirmó la obstrucción en 7 casos. Cinco casos correspondieron a obstrucción pieloureteral, una bilateral. Un caso tenía un riñón en herradura con displasia multicistica del lado izquierdo y otro era portador de un doble sistema izquierdo con hidronefrosis masiva del hemiriñón inferior. En el caso del rínón en herradura si bien se diagnosticó la masa quística izquierda no se detectó el puente que unía los 2 riñones. En 2 casos no había obstrucción: un enfermo tenía reflujo vésico ureteral masivo y el otro dilatación urétero piélica bilateral no obstructiva, secundaria a imperforación anal con gran recto retrovesical lleno de meconio.

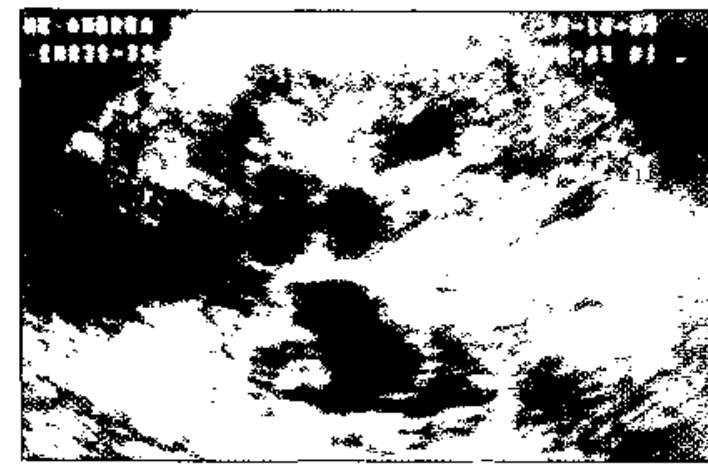

Figura L. Leografía fetal que muestra tiñón hidronefiótico en que destaca una gran pelvis

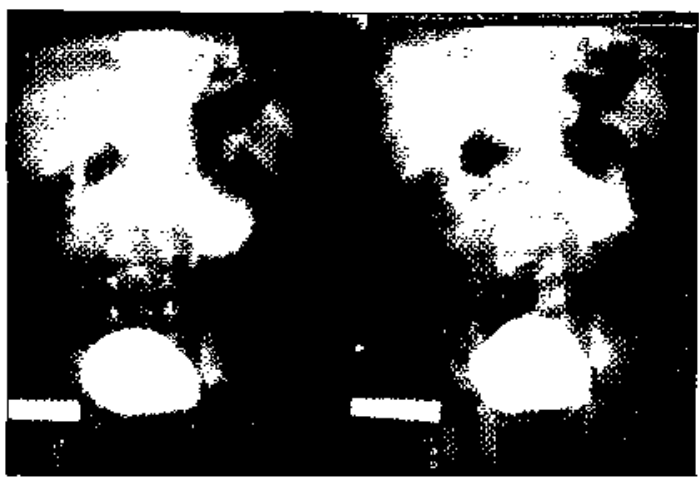

Figura 2. Pielogtatía post operatoria ds obstrucción pielourcteral corregida. Iiste rifón estaba excluido en la piclografía preoperatona de recien nacido.

En un caso, informado como tumor renal izquierdo, en realidad se comprobó doble sistema con ureterocele ectópico obstructivo y displasia renal severa del heminiñón superior. cuyo volumen estaba aumentado por microquistes, que fueron interpretados como masa tumoral.

Nueve niños se operaron. No se operó la vía urinaria del niño que presentaba dilatación ureteropiélica sin obstrucción con imperforación anal baja.

Los resultados de la cirugía neonatal fueron buenos, es especial en los casos de obstrucción pieloureteral en que se practicó plastía eliminando la unión obstruida, anastomosando la pelvis al uréter (plastía de Anderson-Hynes). No se usó tutor ni nefrostomia en ninguna de las plastias pieloureterales.

En un caso con obstrucción pieloureteral severa el rinón estaba excluido en el pre-operatorio. Hubo recuperación de él en la pielografía de control al año de operado (figura 2).

En el caso del uréterocele se practicó heminefrectomía superior y ureterectomía. El uréterocele se abandonó colapsado en vejiga.

Se realizó una heminefrectomía inferior en el caso de obstrucción de hemirinón inferior dado 
el compromiso severo de la corteza renal.

El riñón multicístico se extirpó seccionando el puente del riñón en herradura.

En el caso del reflujo vésicoureteral masivo se hizo vesicostomía inicial por el gran diámetro de los uréteres, lo que aconsejaba diferir el neoimplante uréterovesical.

Todos los enfermos se controlaron pielográficamente entre los 6 meses y el ax̃o de operados. Los controles ecográficos fueron en el post operatorio inmediato, a los 3 meses y al año.

Hubo complicaciones en el caso del reflujo vésicoureteral masivo que se trató con vesicostomía inicial. Esta hizo prolapsos a repetición de difícil reducción, lo que obligó a reimplantar los dos uréteres a los 3 meses. Uno de los reimplantes falló por no modelar (afinar) el urêter, lo que requirió volver a reimplantarlo, con modelado, a los 3 años (figura 3).

\section{COMENTARIO}

Esta serie clínica de malformaciones urológicas diagnosticadas en forma antenatal, si bien reducida, muestra algunas características interesantes.

El diagnóstico más frecuente fue el de abstrucción pieloureteral. Este trastorno, salvo que se asocie precozmente a una masa palpable o infección urinaria, suele diagnosticarse tardiamente en el lactante con infecciones urinarias a repetición o en riños mayores por hematuria a raiz de un traumatismo. El riñón obstruido del niño mayor a veces tiene dafno severo y no ha dado sintomas. Es por eso muy útil poder detectar esta enfermedad en forma antenatal, lo que da al riñón mayor capacidad de recuperación y crecimjento evitando progresión del daño por infección o por la misma hidronefrosis.

La ecografía prenatal en nuestras manos fue bien sensible en diagnosticar hidronefrosis (todos los casos) pero poco discriminativa en el diagnóstico fino, anatómico. Es así como no detectó el doble sistema en una hidronefrosis, $y$ tampoco se diagnosticó un riñón en herradura. En el caso del uréterocele ectópico el ecografista diagnosticó tumor renal que en realidad correspondia a un riñón con displasia quística de quistes pequerios. Creemos que en esto influyen las limitaciones del método ecográfice y el hecho que los ecografistas obstétricos no son especialistas en las innumerables variantes que presentan las malformaciones urológicas congénitas. El trabajo en equipo de los diferentes especialistas puede mejorar mucho el rendimiento del método, sobre todo en cuanto a evaluar la función y el grado de daño renal que se ha producido, el cual varia en distintos pacientes con una misma malformación.

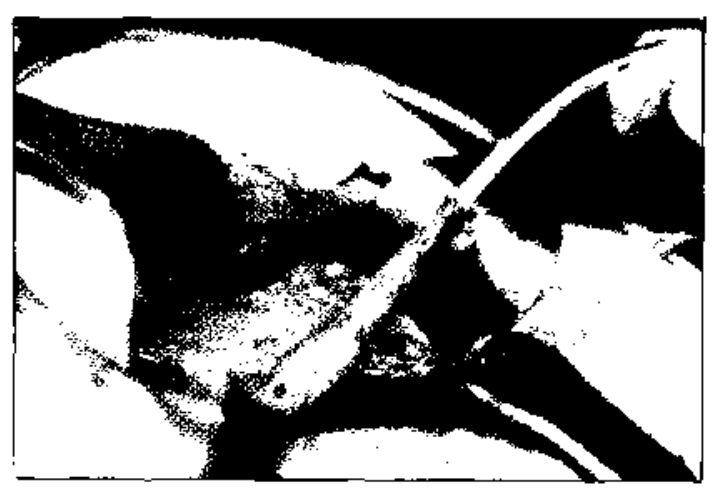

Figura 3. Uréter reimplantado a los 2 meses de edad que requirió reoperación por reflujo vésicoureteral residual debido al gran diámetro ureteral.

En ninguna de las plastias pieloureterales se dejó tutor o nefrostomía usando drenaje de Penrose a la fosa renal por $\mathbf{4 8}$ horas. No estamos contra el uso de tutor y nefrostomia pero pensamos que el progreso de los materiales de sutura los hacen menos necesarios y simplifican el manejo postoperatorio. Se han comunicado buenos resultados por grupos que siguen esta conducta. 3,4

Interesante es comentar la cirugía del caso del uréterocele ectópico. En el sólo se hizo una heminefrectomía superior derecha abandonando el uréterocele pequeño y vacío en la vejiga, to que simplificó la cirugía y disminuyó el sangramiento operatorio. Los controles postoperatorios pielográficos y cistográficos fueron satisfactorios. La técnica de abandonar el uréterocele pequeño colapsado en vejiga la hemos usado en varios casos sin mayores problemas.

Un enfermo presentó una hidronefrosis bilateral antenatal secundaria a obstrucción al vaciamiento vesical y ureteral por un fondo de saco rectal ciego lleno de meconio. Este tipo de problemas se han descrito en el último tiempo y basta con la colostomía o la solución de la imperforación anal para que la hidronefrosis mejore por sí sola. ${ }^{5}$

\section{RESUMEN}

Se presentan 10 casos de diagnóstico antenatal urológico y su evolución posterior. La ecografía en nuestras manos fue muy sensible en el diagnóstico de obstrucción no asi en el diagnóstico fino de la afección causal. Ello pensamos mejorará con mayor experiencia. Los resultados de la cirugía neonatal por obstrucción pieloureteral ( 6 riñones) fueron buenos, recuperándose incluso un riñón excluido en la pielografía. No se usó nefrostomia ni tutor en la pieloplastia. También evolucionaron bien los casos que requirieron 
heminefrectomía superior, ${ }^{1}$ y nefrectomia con sección del puente de riñón en herradura. Sólo una vesicostomia temporal requirió de cirugia posterior al fallar por presentar prolapso grave. No se operó y mejoró en forma espontánea, al hacer una colostomía, un caso con hidronefrosis bilateral a comprensión vesical por el recto ciego de una imperforación anal.

\section{AGRADECIMIENTOS}

Se agradece a la Dra. Colomba Norero $V$. quién revisó la redacción de este trabajo.

\section{REFERENCIAS}

1. Martin, J.J., Taylar, E.S.: Diagnosis of bulateral hydronephrosis in utero by ultrasonography Urology, 17: 272, 1981 .
2. Badlont, G., Abroms, H., Kumari, S.: Diagnosis of fecal hydronephrosis in utero using ultrasound. Uralogy, 16: 315,1980 .

3. Bassam, B. Belman, A.B.: Ureteropelvic junction obstruction in newborns and infants. I liol 128: $770,1982$.

4. King, L.R., Coughlin, P., Bioch, E.C., Bowie, I.D., Ansong, $K$, Hanna. M.K.: The case for inmediate pyeloplasty in the neonate with ureteropelvic junction obstruction. J Urot 132: $725,1984$.

5. Munn, R., Schillinger, J.F.: Urologic abnormalities found with imperforatc anus. Urology 2I: 260 , 1983.

6. Guest, G.. Gacoin, F.: Resultants d'une enquete sur le depistage ante-natal des malformations renourinaires. Seminaire de Nephrologie pediatrique $26-27$ mars 1984, París.

7. Merreweli, C.: Practical abdominal ultrasound, Ed. London, Heinemann, 1978.

8. Ketalis, P., King. L.R., Belman. B.: Clinical pediatric urology. Ld. 2nd Saunders, 181. 1985. 